\title{
SHORT COMMUNICATION \\ Physical Basis of Warkusz's Equation for Polycrystalline Metallic Conductivity
}

\author{
C. R. PICHARD, A. J. TOSSER and C. R. TELLIER \\ Laboratoire d'Electronique Université de Nancy-I, C.O. 140-54037 Nancy Cedex (France)
}

(Received May 10, 1979)

A new grain-boundary scattering model has been recently proposed by Warkusz ${ }^{1}$ but the physical basis does not seem realistic since grain-boundaries are represented by cylindrical scatterers. ${ }^{2}$ The purpose of this paper is to give an acceptable physical basis for the equation of conductivity proposed (Warkusz, ${ }^{1}$ Eq. 8) and to discuss the validity of the corresponding conduction model.

According to Sondheimer's calculations ${ }^{3}$ the conductivity $\sigma_{g}$ of an infinitely thick polycrystalline metal film is

$$
\sigma_{g}=\frac{3}{4} \sigma_{0} \int_{0}^{\pi} \frac{\lambda(\theta)}{\lambda_{0}} \cdot \sin ^{3} \theta \cdot \mathrm{d} \theta
$$

in spherical polar coordinates, where

$\sigma_{0}$ is the bulk conductivity

$\lambda_{0}$ the electronic mean free path in the bulk material

$\lambda(\theta)$ an electron mean free path which takes into account the background and the grain boundary scatterings.

It is assumed that the effect of grain-boundaries can be described by a series $S_{x}$ of randomly distributed scattering planes perpendicular to the applied electric field $E$ (along the $x$-axis) and a series $S_{y}$ of randomly distributed scattering planes, with the same average spacing $D$, parallel to the electric field and perpendicular to the previous ones. Both series determine scattering points on a given direction of electron velocity $V$; the average distance between the scattering points due, respectively, to $S_{x}$ and $S_{y}$ is

$$
\begin{aligned}
& d_{x}=D|\cos \phi|^{-1}|\sin \theta|^{-1} \\
& d_{y}=D|\sin \phi|^{-1}|\sin \theta|^{-1}
\end{aligned}
$$

(See Figure 1).
If the probability $p$ that an electron has travelled a path of length $l$ without being diffusely scattered by grain-boundaries could be analytically described by an exponential law, i.e.

$$
\begin{aligned}
& p_{x}=\exp -\frac{l}{\lambda_{x}} \\
& p_{y}=\exp -\frac{l}{\lambda_{y}}
\end{aligned}
$$

the resultant probability is

$$
P=p_{x} \cdot p_{y}=\exp -\left(\frac{1}{\lambda_{x}}+\frac{1}{\lambda_{y}}\right) l
$$

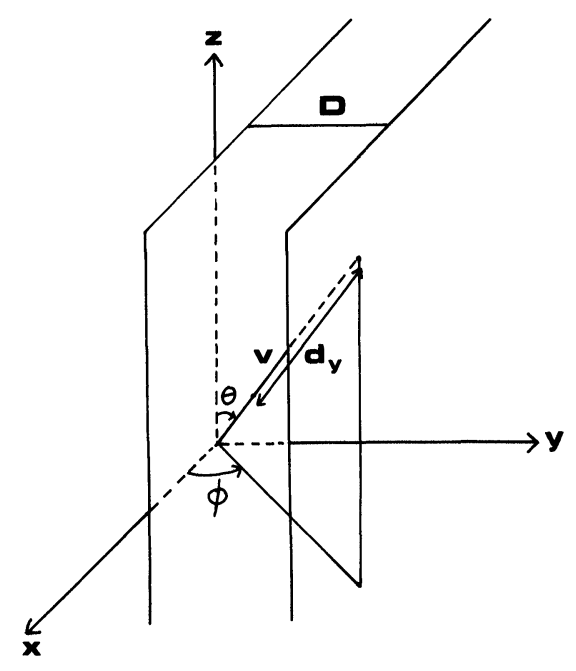

FIGURE 1 Geometry of the Scattering Planes Model. 
where

$$
\begin{aligned}
& \lambda_{x}=D|\cos \phi|^{-1}|\sin \theta|^{-1}\left(\ln \frac{1}{p}\right)^{-1} \\
& \lambda_{y}=D|\sin \phi|^{-1}|\sin \theta|^{-1}\left(\ln \frac{1}{p}\right)^{-1}
\end{aligned}
$$

If grain-boundary scattering and background scattering are independent, $\lambda(\theta)$ could be written as:

$$
\frac{1}{\lambda(\theta)}=\frac{1}{\lambda_{0}}+\frac{1}{\lambda_{x}}+\frac{1}{\lambda_{y}} \text {. }
$$

Hence Eq. (1) becomes

$\sigma_{g}=\frac{3}{4 \pi} \sigma_{0} \int_{0}^{2 \pi} \int_{0}^{\pi}$

$\frac{\sin ^{3} \theta}{1-\frac{\lambda_{0} \ln p}{D}[|\cos \phi|+|\sin \phi|]|\sin \theta|} \mathrm{d} \theta \mathrm{d} \phi$

Assuming that

$$
|\cos \phi|+|\sin \phi| \approx \operatorname{cte}=\frac{4}{\pi}
$$

Eq. (7) becomes

$$
\sigma_{g}=\frac{3}{4} \sigma_{0} \int_{0}^{\pi} \frac{\sin ^{3} \theta}{1-\frac{\lambda_{0} \ln p}{D} \frac{4}{\pi}|\sin \theta|} \mathrm{d} \theta
$$

Introducing

$$
\mu=\frac{D}{\lambda_{0} \cdot \ln \frac{1}{p}} \cdot \frac{\pi}{4}
$$

in Eq. (9) gives:

$$
\sigma_{g} / \sigma_{0}=\frac{3}{4} \int_{0}^{\pi} \frac{\sin ^{3} \theta}{1+\frac{\sin \theta}{\mu}} \mathrm{d} \theta=F(\mu)
$$

which is Warkusz's ${ }^{1}$ Eq. (8) in which $\mu$ has been substituted for $\nu$.

Integration of Eq. (11) gives:

$$
\begin{gathered}
F_{2}(\mu)=\frac{3}{2} \mu\left[\frac{\pi}{4}-\mu+\frac{\pi}{2} \mu^{2}-\frac{\mu^{3}}{\sqrt{\mu^{2}-1}}\right. \\
\left.\left(\frac{\pi}{2}-\operatorname{Arctg} \frac{1}{\sqrt{\mu^{2}-1}}\right)\right]
\end{gathered}
$$

for $\mu>1$, which may be written

$$
\begin{array}{r}
F_{2}(\mu)=\frac{3}{2} \mu\left[\frac{\pi}{4}-\mu+\frac{\pi}{2} \mu^{2}-\frac{\mu^{3}}{\sqrt{\mu^{2}-1}}\right. \\
\left.\operatorname{Arcsin} \sqrt{1-\frac{1}{\mu^{2}}}\right]
\end{array}
$$

and

$$
\begin{aligned}
F_{1}(\mu)=\frac{3}{2} \mu\left[\frac{\pi}{4}-\mu+\frac{\pi}{2} \mu^{2}\right. & +\frac{\mu^{3}}{\sqrt{1-\mu^{2}}} \\
& \left.\ln \frac{\mu}{1+\sqrt{1-\mu^{2}}}\right]
\end{aligned}
$$

for $\mu<1$

Eq. (13) and (14) have been given by Warkusz. ${ }^{1}$

In the limiting cases $\mu \rightarrow 0$ and $\mu \rightarrow \infty$ the approximate expressions of $F(\mu)$ are Eqs (15) and (16) (See Warkusz, ${ }^{1}$ Eqs (9) and (10))

$$
F_{1}(\mu) \approx \frac{3 \pi}{8} \mu \quad \text { for } \mu \ll 1
$$

and

$$
F_{2}(\mu) \approx 1-\frac{9 \pi}{32 \mu} \quad \text { for } \mu \gg 1
$$

whereas the corresponding expressions in the Mayadas-Shatzkes conduction mode ${ }^{4}$ are

$$
f(\alpha) \approx \frac{3}{4 \alpha} \quad \text { for } \alpha \gg 1
$$

and

$$
f(\alpha) \approx 1-\frac{3}{2} \alpha \quad \text { for } \alpha \ll 1
$$

Identifying Eqs (15) and (17), and Eqs (16) and (18), gives

$$
\alpha \cdot \mu=\frac{2}{\pi}=0.636
$$

and

$$
\alpha . \mu=\frac{3 \pi}{16}=0.589
$$

Warkusz $^{1}$ has previously advanced that for

$$
\alpha . \nu=0.62
$$

$F_{1}(\mu)$ and $F_{2}(\mu)$ are alternative expressions for the conductivity ratio in the Mayadas-Shatzkes model $;{ }^{4}$ the theoretical expression of this ratio, $\sigma_{g} / \sigma_{0}$, is 


$$
\sigma_{g} / \sigma_{0}=f(\alpha)
$$

with

$$
f(\alpha)=1-\frac{3}{2} \alpha+3 \alpha^{2}-3 \alpha^{3} \ln \left(1+\frac{1}{\alpha}\right)
$$

However, Eqs (19), (20) and (21) show that marked deviations occur for $\alpha \ll 1$, even if a good fit is obtained in a large range of $\alpha$-values.

Furthermore the relation between $R$ and $P$ deduced from Eqs (21) and (10) must be written as

$$
\frac{R}{1-R}=\ln \frac{1}{p} \times \frac{4}{\pi} \times 0.62
$$

which differs from the relation previously proposed by Warkusz ${ }^{1}$ since the author started from cylindrical boundaries. $^{2}$

Eq. (3) can be written if the effect of scattering planes on the electronic flow along an electron path can be considered as a differential variation and if diffusely scattered electrons do not contribute to the current; this requires that the number of scattering points on any electronic path is high. This condition is satisfied when the width and the length of the film are much larger than the mean grain diameter and if it is assumed that electrons whose velocity vector is near from the $z$-axis do not contribute significantly to the current.

Nevertheless the value of $P$ corresponding to the value of $R \approx 0.4$ experimentally obtained for annealed films of aluminium and zinc ${ }^{5-9}$ seems low (Table I) and it could be wise to examine the validity of the last above assumption.

We will attempt to emphasize this point in a future paper, when a convenient tool of calculation will be available.
TABLE I

Experimental values of $P$ and $R$ obtained for annealed films of aluminium and zinc.

\begin{tabular}{lll}
\hline $\mathrm{R}$ & $1-\mathrm{R}$ & $\mathrm{P}$ \\
\hline 0.1 & 0.9 & 0.8687 \\
0.2 & 0.8 & 0.7286 \\
0.3 & 0.7 & 0.5811 \\
0.4 & 0.6 & 0.4298 \\
0.5 & 0.5 & 0.2817 \\
0.6 & 0.4 & 0.1495 \\
0.7 & 0.3 & 0.0520 \\
0.8 & 0.2 & 0.0063 \\
0.9 & 0.1 & 0.0000112 \\
\hline
\end{tabular}

The conductivity equation proposed by Warkusz can be considered as derived from a representation of grain-boundaries by a bidimensional array of scattering planes. Quantitative agreement is obtained with Mayadas-Shatzkes equations.

\section{REFERENCES}

1. F. Warkusz, Electrocomp. Sc. Tech., 5, 197 (1978).

2. C. R. Tellier and A. J. Tosser, Private communication (1978).

3. E. H. Sondheimer, Adv. Physics, 1, 1 (1952).

4. A. F. Mayadas and M. Shatzkes, Phys. Rev. B, 1, 1382 (1970).

5. C. R. Tellier and A. J. Tosser, Thin Solid Films, 37, 207 (1976).

6. C. R. Tellier and A. J. Tosser, Thin Solid Films, 43, 261 (1977).

7. C. R. Tellier et A. J. Tosser, Le Vide, Suppl., 189, 25 (1978).

8. C. R. Tellier, Thin Solid Films, 51, 327 (1978).

9. C. R. Tellier, Vacuum, 28, 321 (1978). 

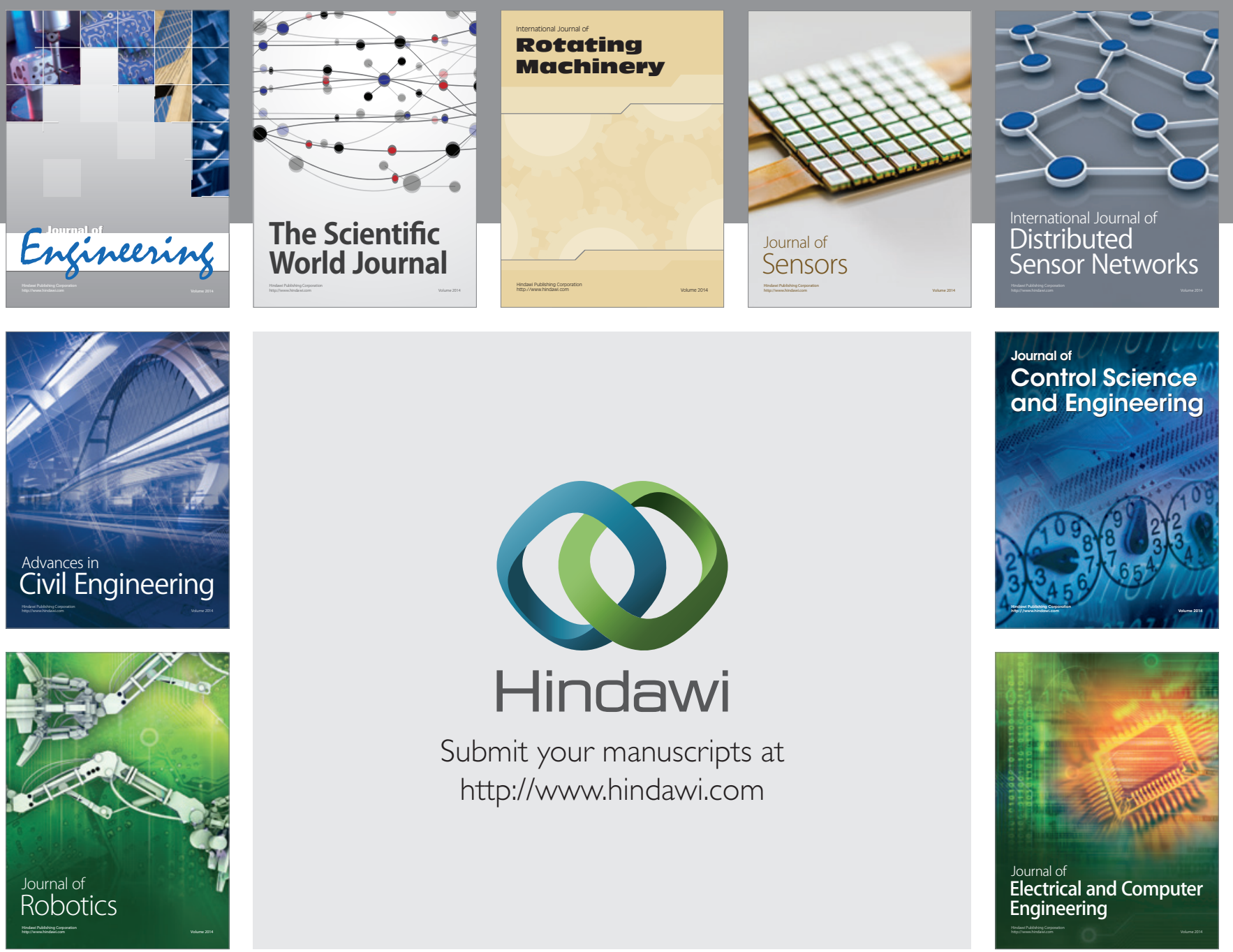

Submit your manuscripts at

http://www.hindawi.com
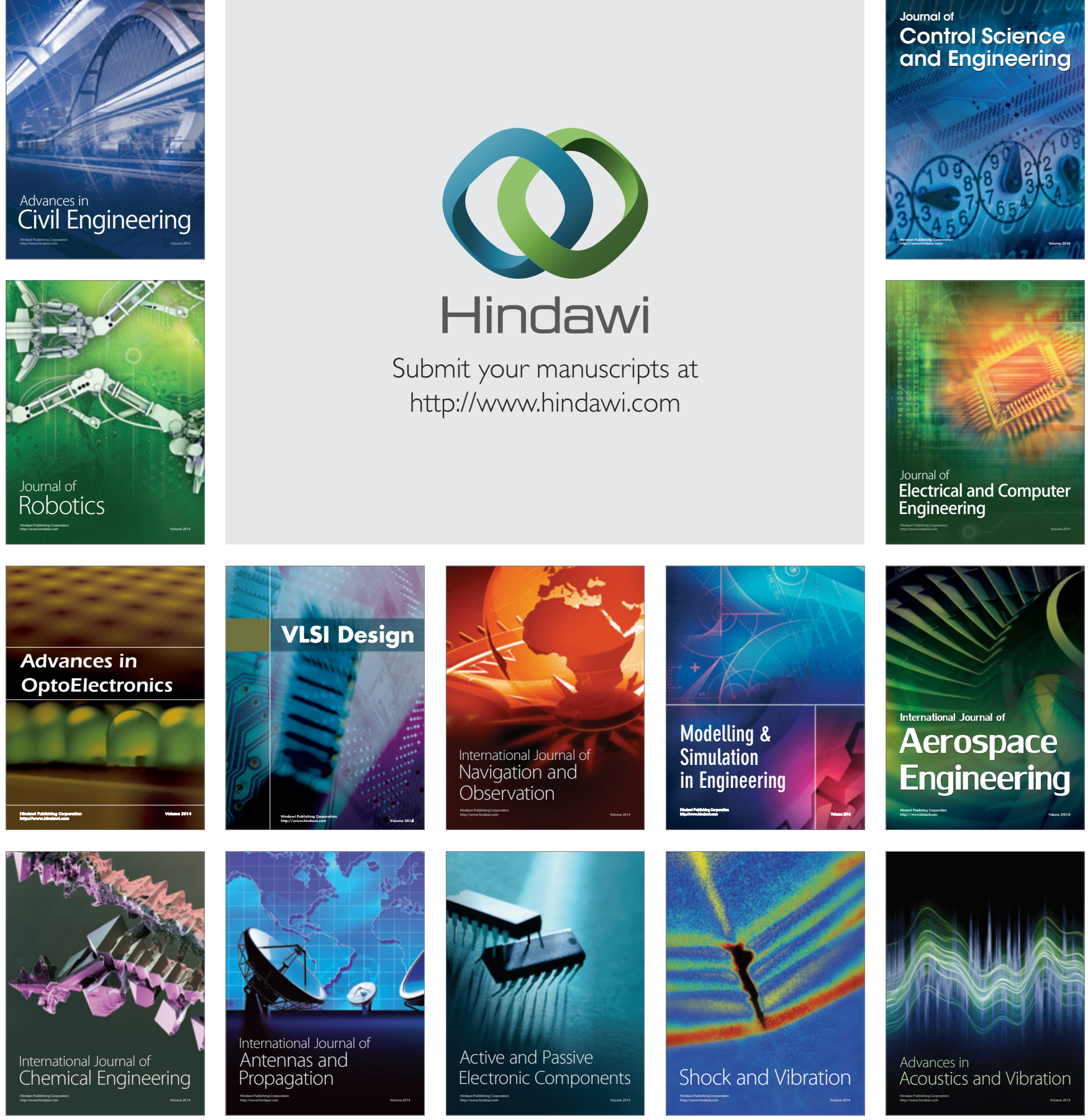